\title{
A simulation model for generation of multiple uncorrelated Rayleigh fading waveforms
}

\section{Vanderlei Aparecido da Silva}

COPEL (Companhia Paranaense de Energia) - Electrical Energy

Company for Generation, Transmission, Distribution and

Commercialization,

R. José Izidoro Biazetto, 158 - Bloco C, s.06 81200-240,

Curitiba, Paraná, Brazil

Fax: +55-41-3234-6090

E-mail: vanderlei.silva@copel.com

\section{Taufik Abrão*}

Electrical Engineering Department, State University of Londrina (DEEL-UEL),

Rod. Celso Garcia Cid - PR445, s/n, Campus Universitário,

P.O. Box 6001, 86051-970, Londrina, PR - Brazil

Fax: +55-43-3371-4790

E-mail: taufik@uel.br

E-mail: taufik.abrao@gmail.com

\section{Paul Jean E. Jeszensky}

Laboratório de Comunicações e Sinais,

Department of Telecommunications and Control Engineering,

Escola Politécnica of University of São Paulo (LCS-PTC-EPUSP),

Av. Prof. Luciano Gualberto, tr. 3, 158,

Cidade Universitária, São Paulo, SP, 05508-900, Brazil

Fax: +55-11-3091-5128

Fax: +55-11-3091-5718

E-mail: pjj@1cs.poli.usp.br

\begin{abstract}
This paper presents a new discrete-time ergodic simulation model for the efficient generation of multiple time uncorrelated Rayleigh waveforms. It uses the inverse discrete Fourier transform (IDFT) to generate the transmission coefficients with only one random number generator. The model and its statistical characteristics are derived as a function of the channel power spectral density (PSD). Irrespective of the channel PSD shape, the proposed model will be an ergodic complex random process with correct ensemble averages.
\end{abstract}

Keywords: Rayleigh fading; channel modelling; Rayleigh waveforms; uncorrelated fading. 
Reference to this paper should be made as follows: Aparecido da Silva, V., Abrão, T. and Jeszensky, P.J.E. (2011) 'A simulation model for generation of multiple uncorrelated Rayleigh fading waveforms', Int. J. Satellite Communications Policy and Management, Vol. 1, No. 1, pp.77-102.

Vanderlei A. da Silva received his BS in Electrical Engineering from UEL - State University of Londrina, Brazil in 2002 and his MSc in Electrical Engineering from EPUSP - Escola Politécnica of University of São Paulo, Brazil in 2004. His research interests include propagation models, channel simulators, and channel estimation methods for MIMO and UWB systems. Since 2006 he is with COPEL - Electrical Energy Company, Paraná, Brazil as a Development Engineer in power energy applications.

Taufik Abrão received his BS, MSc and PhD, all in Electrical Engineering from EPUSP - Escola Politécnica of University São Paulo, Brazil, in 1992, 1996, and 2001, respectively. He is currently an Associate Professor at the Electrical Engineering Department of UEL - State University of Londrina, Brazil. From 2007 to 2008 , he was a Visiting Professor at TSC/UPC Department of Signal Theory and Communications, Universitat Politécnica de Catalunya, Barcelona, Spain. His research interests include multi-user detection, MC-CDMA and MIMO systems, heuristic and optimisation aspects of multiple access systems and networking. He is the author or co-author of more than 70 research papers published in specialised periodicals and key conferences in the area of wireless communication and networking.

Paul Jean E. Jeszensky received his BS, MS and PhD, all in Electrical Engineering from EPUSP - Escola Politécnica of University of São Paulo, Brazil, in 1972, 1981, and 1989, respectively. Since 1990 he has been with EPUSP where he is a Full Professor and Researcher in Communication Systems. He was Visiting Professor at UPC - Universitat Politécnica de Catalunya, Barcelona, Spain in 1995 and at TUB - Technical University of Budapest, Hungary in 2001. He is the author of the book Sistemas Telefônicos (in Portuguese), Editora Manole, 2003 and his current research interests include CDMA systems, multi-user detection, code sequences analysis and related topics.

\section{Introduction}

Statistical modeling of mobile radio channels is often used for analysis and design of wireless communication systems. Some of these mathematical channel models have been accomplished in computers and used as an important tool to simulate signal envelope variations for mobile-radio environment analysis. As an example, Monte Carlo simulation of wireless communication system under fading channel requires an effective mathematical channel model which can efficiently reproduce the main statistical properties of the physical channel in a computer. Effectiveness and efficiency have been motivation for a large number of channel simulator works in the literature in the last 40 years. 
The scattering propagation model of Clarke (1968) has been the base for some widely used fading channel simulators, such as the Jakes (1974) and Smith (1975) models. The former uses a sum of sinusoids to obtain the Rayleigh transmission coefficients, whereas the latter uses a frequency domain filtering of two complex Gaussian random processes.

The Jakes model, as originally presented, is not able to reproduce some important statistical properties found in a physical mobile radio channel. The Smith model can reproduce them, but requires a high computational effort. Therefore, several works with analysis and improvement for both models have been proposed (Dent et al., 1993; Zheng and Xiao, 2003; Xiao et al., 2002; Pop and Beaulieu, 2001; Young and Beaulieu, 2000; Wang and Pätzold, 2003; Iqbal et al., 2009; Alimohammad et al., 2009). In particular, the Jakes model became statistically correct (from the point of view of ensemble averages) and wide-sense stationary (WSS) with the improvement proposed in Zheng and Xiao (2003). On the other hand, a modification in the Smith model algorithm, proposed in Young and Beaulieu (2000), reduced its computational effort by half, keeping the correct statistical properties of the model. The authors in Alimohammad et al. (2009) propose improvements on sum-of-sinusoids-based channel model for wireless radio propagation. The model uses random walk processes instead of random variables (RVs) for some of the sinusoid parameters of a time-correlated Rayleigh and Rician fading channels. Another method for the generation of complex Gaussian stochastic processes is the spectral representation described in Shinozuka and Deodatis (1991) and used by Kodzovi and Abdi (2003) for fading channels simulation (in its Section 2).

This paper proposes a new discrete-time transmission coefficients model for the generation of multiple time uncorrelated Rayleigh waveforms. From a mathematical point of view, the proposed model can reproduce the main statistical properties of a mobile physical channel with every power spectral density (PSD) shape. From performance point of view, it is suitable for applications which require simulation with high number of samples, because it presents fast computational response.

The model was derived as a function of the channel PSD in order to enable channel simulation with other PSD than the well known U shaped. Hence, models based on the Clarke channel PSD can be considered as a particular case of this model. All statistical characteristics were derived as a function of the channel PSD. As a result, irrespective of the channel PSD shape, the transmission coefficients from the model are a complex random process with correct ensemble averages, ergodic, and with Rayleigh envelope and uniform phase angle, which are properties of a physical channel. These are important characteristics of a channel simulator when the PSD does not follow the traditional Clarke and Aulin shapes. In addition, to perform a real-time uncorrelated channel, from the model point of view, every two sample functions are time uncorrelated. All statistical properties were also verified by computational simulations.

The proposed model allows generate the transmission coefficients using only one random number generator. Initially, a complex random process is created within the frequency domain, and then the IDFT is applied to compute the transmission coefficients in the time domain. In fact, in order to improve computational response, the inverse fast Fourier transform (IFFT) has been implemented in the simulator 
algorithm. But, the performance of IFFT is not consistent for a high and/or not a power of two numbers of samples. Therefore, this work presents an algorithm to handle the number of samples in the model. As a result, the execution time curve for every number of samples $N$ fits the execution time curve for $N$ in steps of powers of two (the best case for IFFT).

The proposed model has been compared with other models in literature to show its effectiveness. Two recent modified versions of the Jakes and Smith models, named as modified Smith model (Young and Beaulieu, 2000) and modified Jakes model (Zheng and Xiao, 2003) were analysed. The results, presented by the authors in Silva et al. (2004) and reproduced here, show that IDFT models require less computational effort than the sum of sinusoids models and show they match the statistical properties of a real channel.

This paper is composed as follows. Section 2 presents a review of the channel mathematical models and its statistical properties. In Section 3 the proposed model is described and analysed. Section 4 presents some computational aspects besides Clarke and Aulin discrete models. Simulations results are presented in Section 5. Finally, Section 6 summarises the main conclusions. Further mathematical details are justified in three appendices. Appendix D lists the main mathematical symbols used in this paper.

\section{Review of channel mathematical models}

Consider the scattering propagation model of Aulin (1979) shown in Figure 1, which is a generalisation of the Clarke (1968) model. At every point, the received signal is composed by a superposition of $M$ plane waves, where the $n$th wave is arriving with an azimuth angle $\alpha_{n}$, an elevation angle $\beta_{n}$ and a carrier phase angle $\phi_{n}$, which are random for different receiving positions. In this model, the transmission coefficient is given by (Aulin, 1979)

$$
c(t)=\frac{1}{\sqrt{M}} \sum_{n=1}^{M} e^{j\left(\omega_{n} t+\phi_{n}\right)}
$$

where $1 / \sqrt{M}$ is the common amplitude of the $M$ waves, $\omega_{n}=\omega_{m} \cos \alpha_{n} \cos \beta_{n}$ is the Doppler frequency shift of the $n$th wave, $\omega_{m}=2 \pi f_{m}, f_{m}=v / \lambda$ is the maximum Doppler frequency shift, $\lambda$ is the wave length of $\omega_{c}$, and $v$ the mobile constant speed.

If $\alpha_{n}$ is uniformly distributed in $[0,2 \pi)$, provided that $M$ is sufficiently large and $\phi_{n}, \alpha_{n}$ and $\beta_{n}$ are independent RVs and also independent of each other, then $c(t)$ is a complex Gaussian random process in conformity with the central limit theorem CLT) with the following ensemble averages (Aulin, 1979):

$$
\begin{aligned}
& E\{c(t)\}=0 \\
& R(\tau)=E\left\{c^{*}(t) c(t+\tau)\right\}=\int_{-\pi}^{\pi} J_{0}\left(\omega_{m} \tau \cos \beta\right) p_{\beta}(\beta) d \beta
\end{aligned}
$$




$$
\begin{aligned}
g(\tau) & =E\{\operatorname{Re}\{c(t)\} \operatorname{Re}\{c(t+\tau)\}\} \\
& =E\{\operatorname{Im}\{c(t)\} \operatorname{Im}\{c(t+\tau)\}\}=\frac{1}{2} R(\tau) \\
h(\tau) & =E\{\operatorname{Re}\{c(t)\} \operatorname{Im}\{c(t+\tau)\}\}=0
\end{aligned}
$$

where $J_{0}($.$) is the Bessel function of the first kind and zero order, and p_{\beta}(\beta)$ is the probability density function (pdf) of the RV $\beta$.

Figure 1 Scattering propagation model

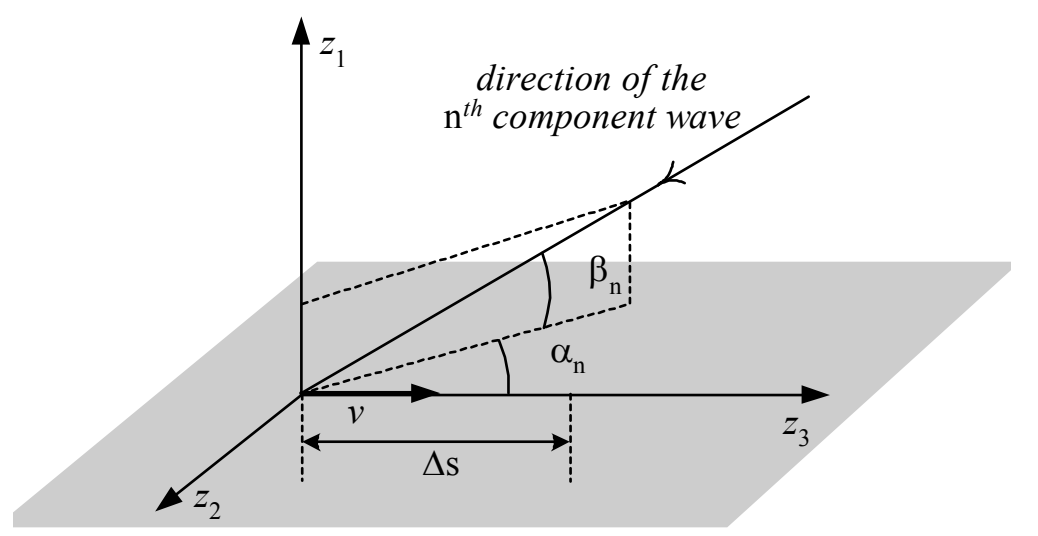

From (2), one can conclude that $c(t)$ is WSS (assuming that $p_{\beta}(\beta)$ is time invariant); $|c(t)|$ (at every time $t$ ) is a Rayleigh RV; $\angle c(t)$ is a uniform RV in the interval $[0,2 \pi$ ) and the real and imaginary parts of $c(t)$ are independent random processes. Moreover, it can be shown that all time averages of every sample function of $c(t)$ are equal to the correspondent ensemble averages, i.e., $c(t)$ is ergodic.

The PSD of $c(t)$ is $F\{R(\tau)\}$. In general, to carry out this Fourier transform is not straightforward. However, considering

$$
p_{\beta}(\beta)= \begin{cases}\frac{\cos \beta}{2 \sin \beta_{\max }}, & |\beta| \leq \beta_{\max } \leq \frac{\pi}{2} \\ 0, & \text { elsewhere }\end{cases}
$$

which can be a realistic pdf for small $\beta_{\max }$, the resulting PSD is given by (Aulin, 1979)

$$
S_{c}(f)=\left\{\begin{array}{lr}
0, & |f|>f_{m} \\
A, & B \leq|f| \leq f_{m} \\
\frac{A}{\pi}\left(\frac{\pi}{2}-\sin ^{-1}\left(\frac{2 \cos ^{2} \beta_{\max }-1-\left(f / f_{m}\right)^{2}}{1-\left(f / f_{m}\right)^{2}}\right)\right), \\
|f| \leq B
\end{array}\right.
$$


where $A=\left(2 f_{m} \sin \beta_{\max }\right)^{-1} ; B=f_{m} \cos \beta_{\max }$ and $\beta_{\max }$ is the highest elevation angle.

In the Clarke (1968) model, the component waves are traveling horizontally, i.e., $\beta_{n}=0$, for every $n$, and $p_{\beta}(\beta)=\delta(\beta)$, where $\delta($.$) is the Dirac delta function.$ Consequently,

$$
\begin{aligned}
& R(\tau)=J_{0}\left(\omega_{m} \tau\right) \\
& S_{c}(f)=F\left\{J_{0}\left(\omega_{m} \tau\right)\right\}=\frac{1}{\pi f_{m} \sqrt{1-\left(f / f_{m}\right)^{2}}}, \quad|f| \leq f_{m}
\end{aligned}
$$

The remaining ensemble averages in (2) do not change.

Additionally, the level-crossing rate (LCR) $N_{R}$, and the average fading duration (AFD) $\tau_{R}$, in a Rayleigh fading channel are given by (Jakes, 1974)

$$
\begin{aligned}
& N_{R}=\sqrt{2 \pi} f_{m} \rho e^{-\rho^{2}} \\
& \tau_{R}=\frac{e^{\rho^{2}}-1}{\sqrt{2 \pi} f_{m} \rho}
\end{aligned}
$$

where $\rho=l / l_{r m s}, l$ is a specified signal level, $l_{r m s}=\sqrt{2 \sigma^{2}}$, and $2 \sigma^{2}$ is the mean square value of $c(t)$.

\section{Discrete transmission coefficients model}

\subsection{Model}

Let $c_{T}(t)$, the truncated version of the transmission coefficients $c(t)$ (suitable for computational purposes), be given by

$$
c_{T}(t)= \begin{cases}c(t), & -T / 2 \leq t \leq T / 2 \\ 0, & \text { otherwise }\end{cases}
$$

Assuming that the transmission coefficients are ergodic in autocorrelation function (e.g., as in the Clarke and Aulin models), its PSD becomes (Couch, 2006)

$$
S_{c}(f)=\lim _{T \rightarrow \infty}\left(\frac{\left|C_{T}(f)\right|^{2}}{T}\right)
$$

where $C_{T}(f)=F\left\{c_{T}(t)\right\}$. In this case the PSD of $c_{T}(t)$ is given by

$$
S_{T}(f)=\frac{\left|C_{T}(f)\right|^{2}}{T}
$$

where

$$
S_{c}(f)=\lim _{T \rightarrow \infty} S_{T}(f)
$$


Besides, if $S(f)$ is the normalised PSD of $c_{T}(t)$, i.e.,

$$
\int_{-\infty}^{+\infty} S(f) d f=1
$$

then,

$$
S_{T}(f)=2 \sigma^{2} S(f)
$$

Now, let $\varphi(f)$ be the phase angle of $C_{T}(f)$. Then, by using (10) and (12), $C_{T}(f)$ becomes

$$
C_{T}(f)=\left|C_{T}(f)\right| e^{j \varphi(f)}=\sqrt{2 \sigma^{2} T S(f)} e^{j \varphi(f)}
$$

Finally, the truncated version of the transmission coefficients is obtained from

$$
c_{T}(t)=F^{-1}\left\{C_{T}(f)\right\}=\sqrt{2 \sigma^{2} T} F^{-1}\left\{\sqrt{S(f)} e^{j \varphi(f)}\right\}
$$

where $F^{-1}\{\cdot\}$ is the inverse Fourier transform.

If the PSD and $\varphi(f)$ are known, equation (14) shows that it is possible to generate a truncated version of the transmission coefficients for simulation purposes. In this case one can assume that the PSD of $c_{T}(t)$ approximates the channel PSD. This approximation improves by increasing the observation interval $T$. In particular, it will be assumed in this work that $\varphi(f)$ is an independent and uniform RV in the interval $[0,2 \pi)$. As a result, it will be shown that the ensemble averages of the random process $c_{T}(t)$, obtained from (14), are only function of the channel PSD. Besides, the equivalent random processes $\left|c_{T}(t)\right|$ and $\angle c_{T}(t)$ are Rayleigh and uniform in the interval $[0,2 \pi)$, respectively.

In order to achieve computational synthesis, it is necessary to transform (14) in a discrete-time equation. Let $c[n]$ be a complex sequence of length $N$, which represents $c_{T}(t)$ sampled with a sampling period $T_{s}=1 / f_{s}$ seconds. The relationship between $\left|C_{T}(f)\right|$ [which is equal to $\left.\left|F\left\{c_{T}(t)\right\}\right|\right]$ and its frequency sampled equivalent version $|C[k]|=|\operatorname{DFT}\{c[n]\}|$, where DFT $\{$.$\} is the discrete Fourier transform, is given by$ (Oppenheim et al., 1999)

$$
\begin{aligned}
|C[k]| & =\frac{1}{T_{s}}\left|C_{T}\left(k f_{\Delta}\right)\right|=\frac{1}{T_{s}} \sqrt{2 \sigma^{2} T S\left(k f_{\Delta}\right)} \\
& =\sqrt{2 \sigma^{2} \frac{N}{T_{s}} S\left(k f_{\Delta}\right)}=\sqrt{2 \sigma^{2} N S[k]}
\end{aligned}
$$

where $f_{\Delta}=1 / T \mathrm{~Hz}$ is the frequency interval between $k$ th and $(k+1)$ th samples, $T=N T_{s}$ is the observation interval in (9) and

$$
S[k]=\frac{1}{T_{S}} S\left(k f_{\Delta}\right)
$$


Finally, the discrete-time transmission coefficients model is given by

$$
\begin{aligned}
c[n] & =\operatorname{IDFT}\{C[k]\}=\sqrt{2 \sigma^{2} N} \operatorname{IDFT}\left\{\sqrt{S[k]} e^{j \varphi_{k}}\right\} \\
& =\sqrt{\frac{2 \sigma^{2}}{N}} \sum_{k=0}^{N-1} \sqrt{S[k]} e^{j\left(\varphi_{k}+2 \pi k n / N\right)}, \quad n=0,1, \ldots, N-1
\end{aligned}
$$

where $\left\{\varphi_{k}\right\}$ are independent RVs with uniform distribution in the interval $[0,2 \pi)$.

The transmission coefficients synthesis block diagram is shown in Figure 2, where $\varphi[k]=\left\{\varphi_{0}, \varphi_{1}, \ldots, \varphi_{k}, \ldots, \varphi_{N-1}\right\}$.

Figure 2 Proposed model diagram

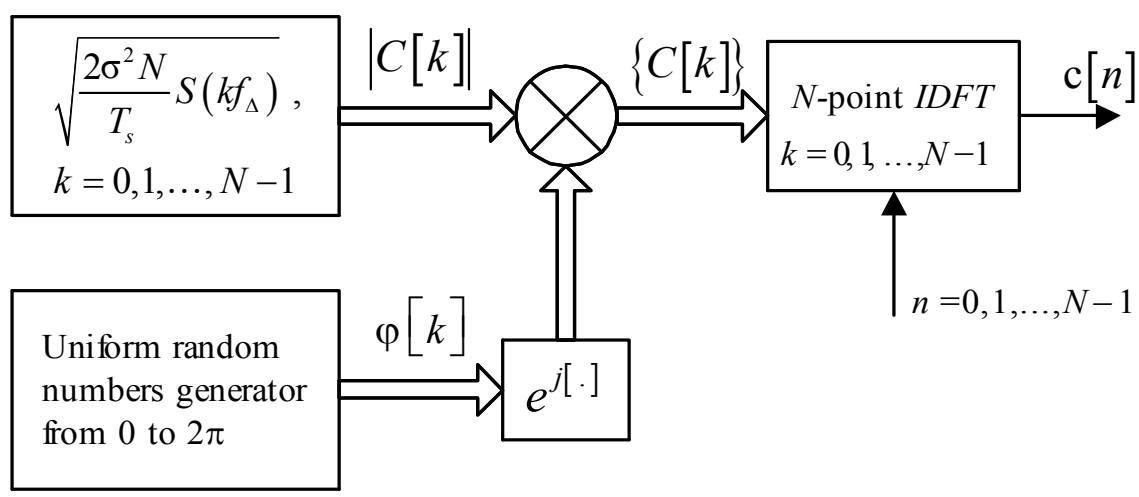

\subsection{Statistical properties}

Equation (18) shows a sum of independent RVs. Hence, from CLT, $c[n]$ is a complex Gaussian random process. The ensemble averages of $c[n]$, computed in Appendix A, are function of the discrete normalised PSD channel $S[k]$, where

$$
\begin{aligned}
& E\{c[n]\}=0 \\
& R[m]=2 \sigma^{2} \operatorname{IDFT}\{S[k]\}, \quad 0 \leq m<N \\
& g[m]=\sigma^{2} \operatorname{Re}\{\operatorname{IDFT}\{S[k]\}\}, \quad 0 \leq m<N \\
& h[m]=\sigma^{2} \operatorname{Im}\{\operatorname{IDFT}\{S[k]\}\}, \quad 0 \leq m<N
\end{aligned}
$$

It should be noted that $g[m]$ (which is equal to $g_{R}[m]$ and $g_{I}[m]$ ) and $h[m]$ are the auto and cross-correlation of the real and imaginary parts, respectively, and $R[m]$ is the autocorrelation function. The range of values for $m$ in (19) can also be $-N / 2 \leq m<N / 2$ ( $N$ even), because $R[m]$ [see the sum in (A2)] is a periodic sequence with period $N$. 
From the statistical averages in (19), it can be concluded that $c[n]$ is WSS. Moreover, the discrete equivalent random processes $|c[n]|$ and $\angle c[n]$ have Rayleigh and uniform distributions in the interval $[0,2 \pi$ ), respectively (Papoulis, 2001).

All time averages of $c[n]$ (computed in Appendix B) for every sample function are equal to the corresponding ensemble averages, irrespective of $S[k]$. As a result, irrespective of the channel PSD shape, the proposed discrete transmission coefficients model $\mathrm{c}[\mathrm{n}]$ is an ergodic random process. Furthermore, the time cross-correlation variance between two sample functions approaches zero as the number of samples or the simulation time increases (Appendix B, item $\mathrm{f}$ ).

\subsection{Model applications}

Considering $S[k]$ representing the PSD of the Aulin model, the ensemble averages for the proposed model can be calculated using (19) with $p_{\beta}(\beta)=\delta(\beta)$ and $N \rightarrow \infty$, as follows

$$
\begin{aligned}
& R[m]=2 \sigma^{2} \int_{-\pi}^{\pi} J_{0}\left(\omega_{m} m T_{S} \cos \beta\right) p_{\beta}(\beta) d \beta \\
& g[m]=\frac{1}{2} R[m] \\
& h[m]=0, \quad \forall m
\end{aligned}
$$

It can be verified that the discrete-time expressions founded in (20) are equivalent to the continuous-time ones in (2).

The same reasoning can be applied when the Clarke scattering propagation model (Clarke, 1968) is used for setting the PSD in the proposed model. From (20) with $p_{\beta}(\beta)=\delta(\beta)$ (waves traveling horizontally) and $-N / 2 \leq m<N / 2$, it follows

$$
\begin{aligned}
& R[m]=2 \sigma^{2} J_{0}\left(\omega_{m} m T_{s}\right) \\
& g[m]=\frac{1}{2} R[m] \\
& h[m]=0, \quad \forall m
\end{aligned}
$$

which are the well-known ensemble averages of the Clarke model.

Figure 3 shows two accomplishments of (19b) [see (A2)] for $-10^{3} \leq m \leq 10^{3}$ and $0 \leq \mathrm{k} \leq 5 \times 10^{4}$, with Aulin and Clarke PSD being compared to (20a) and (21a). The approximation error is very small and is due to the finite range of values used for $k$.

From (21), (A3) and (A5) (when the Clarke model is used for setting the PSD) and from Jakes (1974), it follows that the LCR and AFD for the proposed model are in agreement with (7) and (8) respectively, when $N \rightarrow \infty$. Simulation results, shown in Figure 12, illustrate the above mentioned results. 
Figure 3 Autocorrelation functions for $-103 \leq m \leq 10^{3}, f_{m} T_{s}=0.025, \tau=m T_{s}$ and $2 \sigma 2=1$; (a) $R[\mathrm{~m}]$ from (20a) with $p_{\beta}(\beta)$ from (3), and $\beta_{\max }=40^{\circ}$ versus $R[\mathrm{~m}]$ from (19b) with $S[k]$ from (24) (b) $R[m]$ from (21a) versus $R[m]$ from (19b) with $S[k]$ from (22)

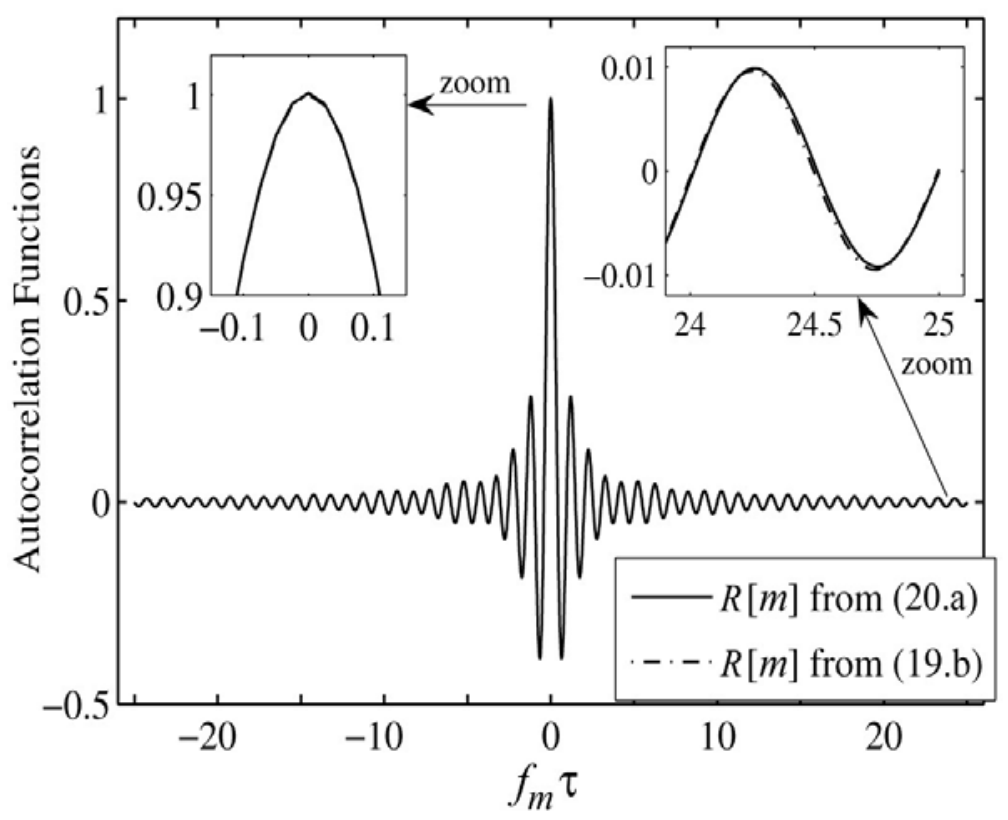

(a)

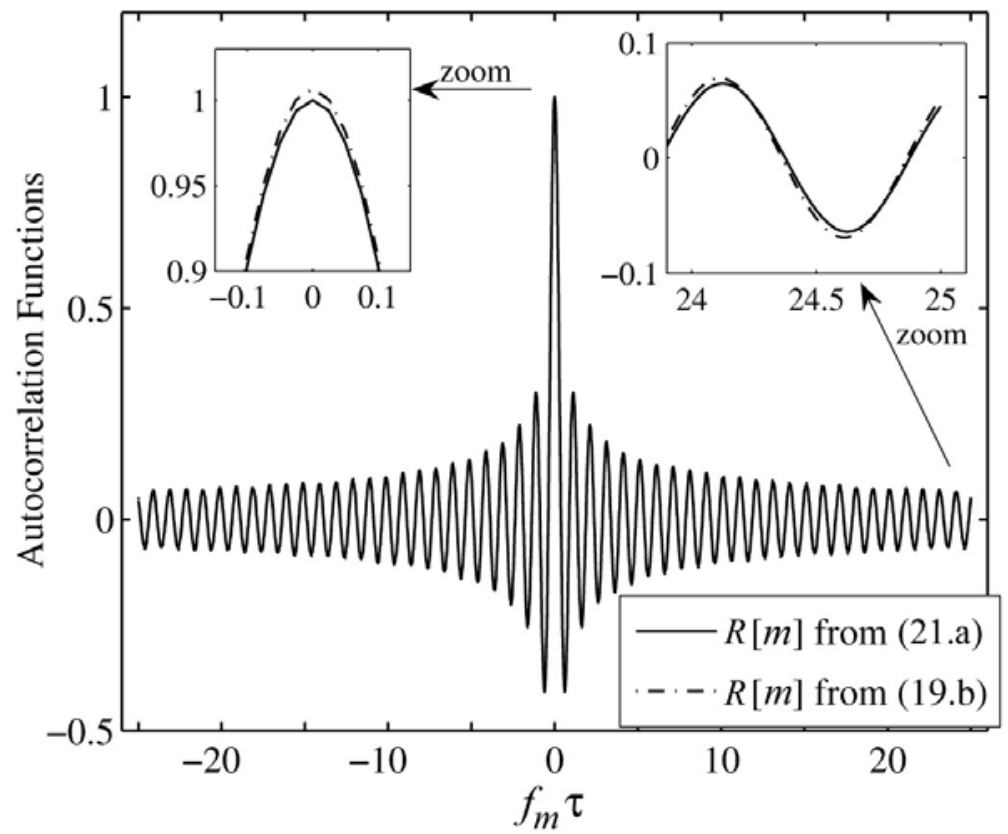

(b) 


\section{Some computational aspects}

\subsection{Using the IFFT}

In order to save execution time, the IDFT in (18) should be computed using some suitable IFFT algorithm. In particular, the Frigo FFT and IFFT algorithms (Clarke, 1968), which are based on the Cooley-Tukey (1965) algorithm, compute the DFT and IDFT more efficiently. These algorithms are used by the MATLAB ${ }^{\circledR}$ package and they can also be found in the $\mathrm{C}$ language.

The execution time for the Frigo algorithm depends on the length $N$ of the sequence. It is fastest when $N$ is a power of two and, besides, it is almost as fast when $N$ is a product of small prime factors. However, it is several times slower when either $N$ has large prime factors or $N$ is a prime number. Thus, before computing the IFFT, in order to improve the algorithm computation time, it is possible to quantize $N$ in a number $N_{t}$, which must be either a power of two, or a number represented by the product of small prime factors. A routine to implement this procedure is illustrated in Figure 4. It should be emphasised that:

- $\quad N_{t} \geq N$ always

- $\quad N_{t}$ is the nearest number to $N$ which is either a power of two or a number with all prime factors $\leq$ factor_min, and where $N_{t}$ is also a multiple of $10^{4}$ (e.g., to decrease the number of searches when $N$ is a large number).

Figure 4 Procedure to transform $N$ in $N t$

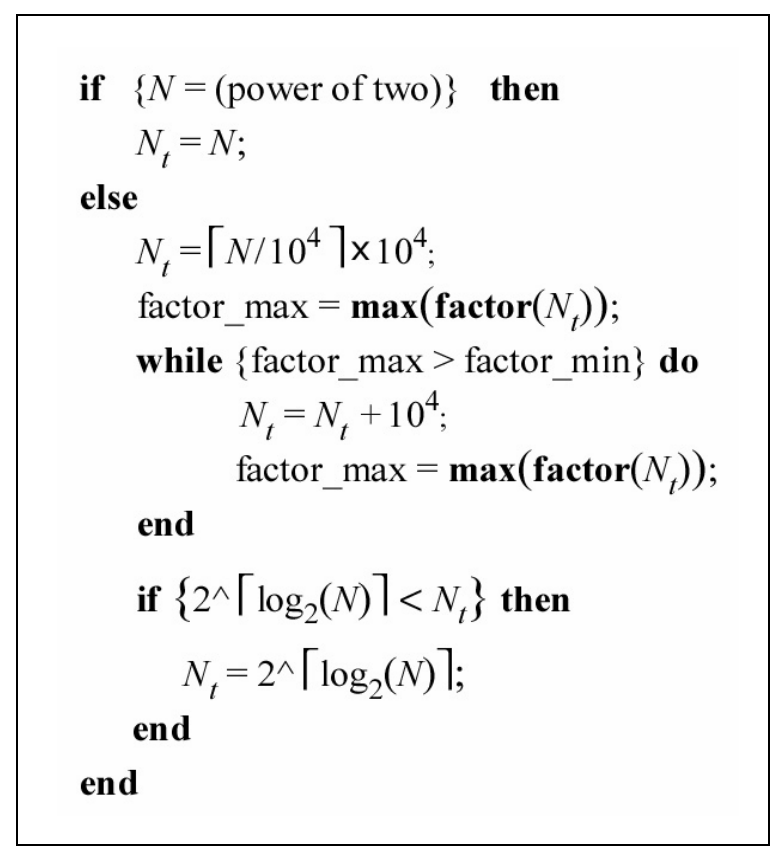

Note: $\max (\mathbf{v})$ returns the largest element in the vector $\mathbf{v}$; factor $(N)$ returns a vector containing the prime factors of $N ;\lceil x\rceil$ rounds $x$ to the nearest integer $\geq \mathrm{x}$. 
In the channel simulator algorithm, the IFFT is applied to an $N_{t}$ length sequence, but the length of the resultant sequence must be $N$. Therefore, $N_{t}-N$ excess samples are nullified subsequent to the IFFT.

A channel simulator algorithm performance, using the procedure in Figure 4, with factor $\min =13$, is illustrated in Figure 5. Note that whenever the value of $N$ is in the considered range, the corresponding execution time is very near the execution time curve for $N$ as power of two (i.e., the best case).

Figure 5 Normalised execution time averaged over 30 simulations with a set of six sample functions

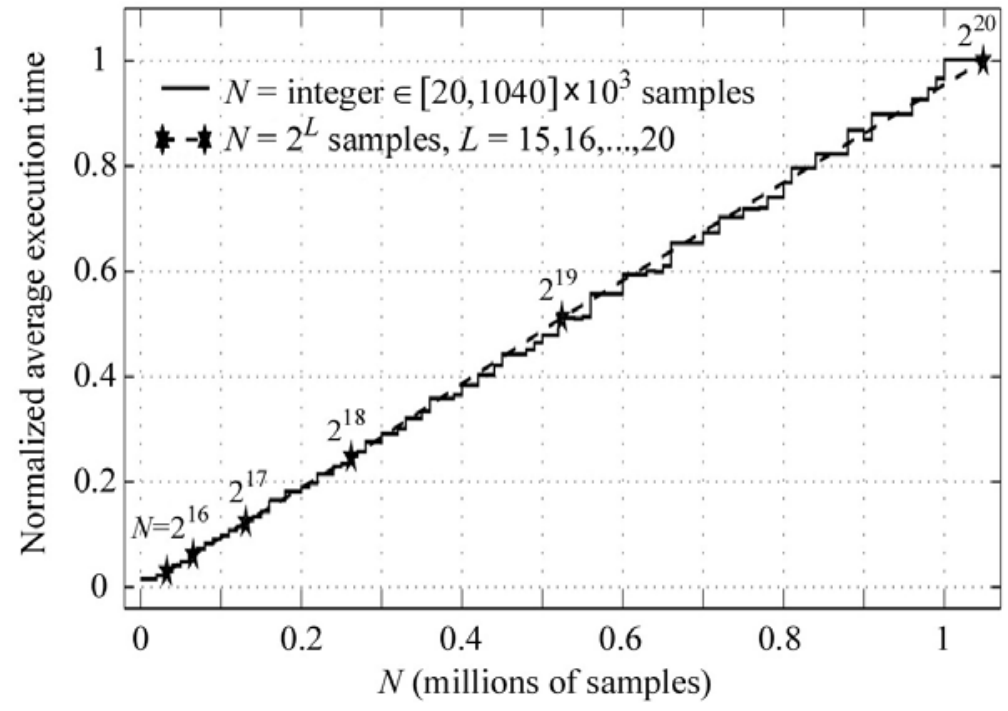

\subsection{Clarke and Aulin PSDs}

The Clarke discrete PSD can be obtained by using (6) and (17), as follows

$$
S[k]=\left\{\begin{array}{lr}
0, & k=0 \\
\frac{1}{T_{s}} S\left(k f_{\Delta}\right), & 1 \leq k<k_{m} \\
N\left(\frac{1}{2}-\frac{1}{\pi} \sin ^{-1}\left(\frac{\left(k_{m}-1\right) f_{\Delta}}{f_{m}}\right)\right), & k=k_{m} \\
0, & k_{m}<k<N-k_{m} \\
S[N-k], & N-k_{m} \leq k \leq N-1
\end{array}\right.
$$

where

$$
k_{m}=\left\lfloor\frac{f_{m}}{f_{s}} N\right\rfloor
$$

and $\lfloor x\rfloor$ rounds $x$ to the nearest integer $\leq x$. In order to assure zero time average [i.e., 
not only when $N \rightarrow \infty$ [see (B1)] but also when $N$ is finite], without loss of generality, it is assumed $S[k=0]=0$. Besides, $S\left[\mathrm{k}=k_{m}\right]$ is found by an interpolation process as described in Appendix C.

With an analogous procedure, the Aulin discrete PSD can be obtained by using (4) and (17) in conformity with

$$
S[k]=\left\{\begin{array}{r}
0, \\
\frac{A}{\pi T_{S}}\left(\frac{\pi}{2}-\sin ^{-1}\left(\frac{2 \cos ^{2} \beta_{\max }-1-\left(\frac{k f_{\Delta}}{f_{m}}\right)^{2}}{1-\left(\frac{k f_{\Delta}}{f_{m}}\right)^{2}}\right)\right. \\
1 \leq k<k_{b} \\
k_{b} \leq k \leq k_{m} \\
\frac{1}{T_{s}} A, \\
0, \\
S[N-k], \\
k_{m}<k<N-k_{m} \\
N-k_{m} \leq k \leq N-1
\end{array}\right.
$$

where $S[0]=0$ again, $k_{m}$ is given by (23) and

$$
k_{b}=\left\lceil\frac{B}{f_{s}} N\right\rceil
$$

and where $\lceil x\rceil$ rounds $x$ to the nearest integer $\geq x$.

In order to obtain $S[k]$ from other theoretical PSD $S(f)$ (e.g., symmetrical or asymmetrical), equally frequency spaced samples are taken from $S(f)$ (considering $0 \leq f<f_{s}$ ) as described in procedures (22) and (24).

\section{Simulation results}

In addition to the complete theoretical analysis shown in this work, the proposed transmission coefficients model has also been exhaustively tested and validated from the numerical standpoint, through several simulations. Figures 6 to 14 present some simulation results obtained from the output of the channel simulator. The proposed model has been tested and compared (Silva et al., 2004) with two other models derived from Clarke PSD: the first one obtained from the Jakes model and defined in Zheng and Xiao (2003), named here as modified Jakes model and the other one, the Smith model defined in Young and Beaulieu (2000), named here as modified Smith model. Figures 6 to 9 present the comparison between the three models. The simulation parameters are: $2 \sigma^{2}=1, f_{m} T_{s}=0.025, N=5 \times 10^{4}$ for all models and eight oscillators for modified Jakes model (as suggested in Zheng and Xiao, 2003). Numerical pdfs were obtained through histograms with 30 columns. The modified Smith model and the proposed model present similar and satisfactory results for all cases, as shown in Figures 6 to 8. It can be verified that the autocorrelation curve (Figure 8 ) of the proposed model fits the reference curve slightly better than the 
modified Smith model. Note also that the modified Jakes model presents some disagreements for all results. This is a direct consequence of the small number of oscillators used in this model. The normalised average execution time in Figure 9 shows that the two IDFT models are approximately three times faster than the modified Jakes model, even considering the number of oscillators as small as eight.

Figures 10 to 14 present some additional simulation results only for the proposed model. All simulations were accomplished with the following parameters: $\beta_{\max }=0^{\circ}$ (Clarke PSD), $\beta_{\max }=40^{\circ}$ (Aulin PSD), $2 \sigma^{2}=1, N=5 \times 10^{4}$ and $f_{m} T_{s}=0.025$. Note that all numerical results are in agreement with the expected theoretical values. Figure 10 shows the cross-correlation of the real and imaginary parts of $c[n]$. In this figure, and in the sequel $\tau=\left(n_{2}-n_{1}\right) T_{s}=m T_{s}$ designates the temporal delay between the samples sequences $c[n]$. The values are close to zero, so they can be considered as time uncorrelated. Figure 11 shows the autocorrelation of $c[n]$ generated with Aulin PSD. Note that this simulation result coincides with the reference value. Figure 12 shows the level crossing rate and AFD considering Clarke PSD. Again, the simulated results and those obtained with equations (7) and (8) coincide. Figure 13 shows the cross-correlation $C_{c}[m]$ of two sample functions generated with Clarke PSD. The results for the Aulin PSD are similar. From these results it can be inferred that in a multipath environment, every two multipath components (e.g., each sample function of $c[n]$ regarded as a multipath component) can be considered time uncorrelated. In this figure, $\sigma_{c}^{2}$ denotes the calculated variance (Appendix B, item f) and agrees with those obtained in Figure 14, which shows the variance of $C_{c}[\mathrm{~m}]$ using Clarke and Aulin PSDs. This figure confirms that $\sigma_{c}^{2}$ approaches zero with increasing $N$.

Figure 6 Probability density functions of a single sample function

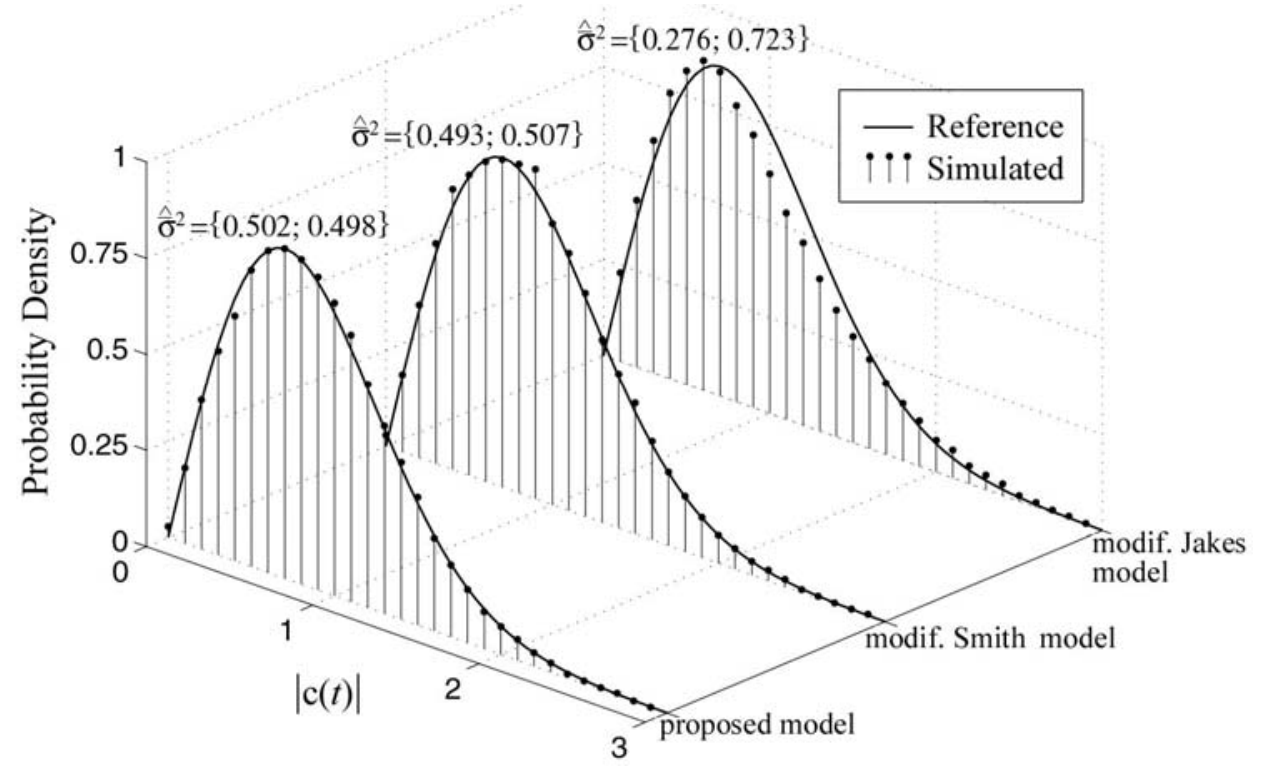

Notes: $\sigma^{2}=\{\operatorname{var}(\operatorname{Re}\{c(t)\}) ; \operatorname{var}(\operatorname{Im}\{c(t)\})\}$. Rayleigh pdf with $\sigma^{2}=0.5$. 
Figure 7 Probability density of phase angle

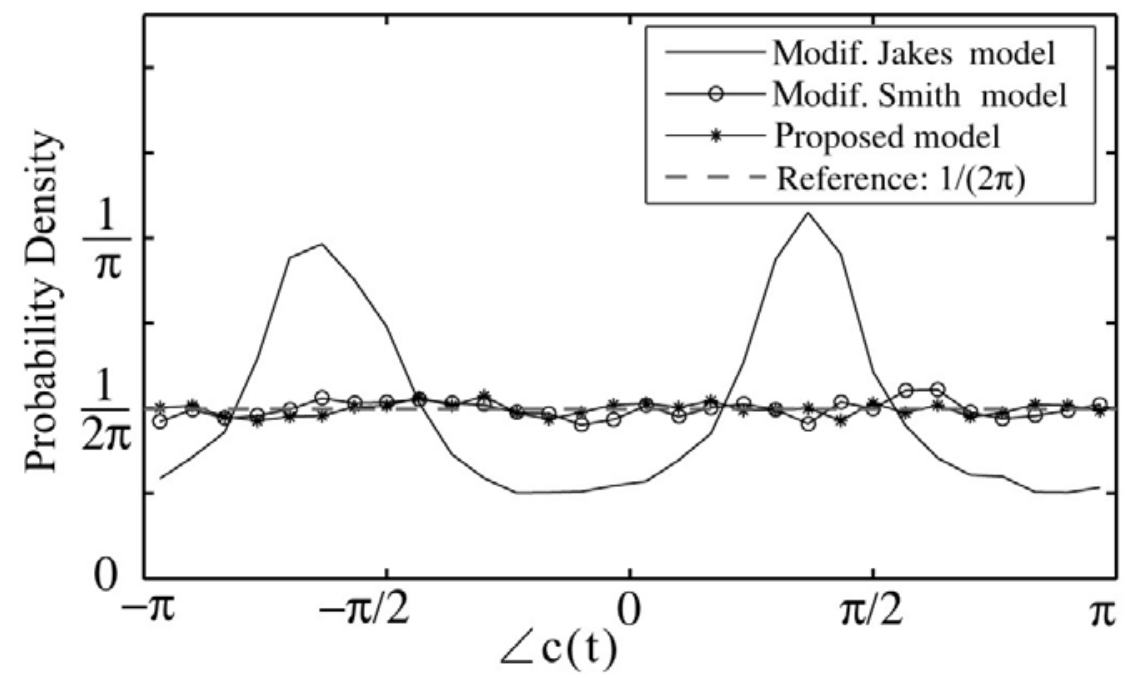

Note: $1 /(2 \pi)$

Figure 8 Time-autocorrelation function; $c[n]$ generated using Clarke PSD

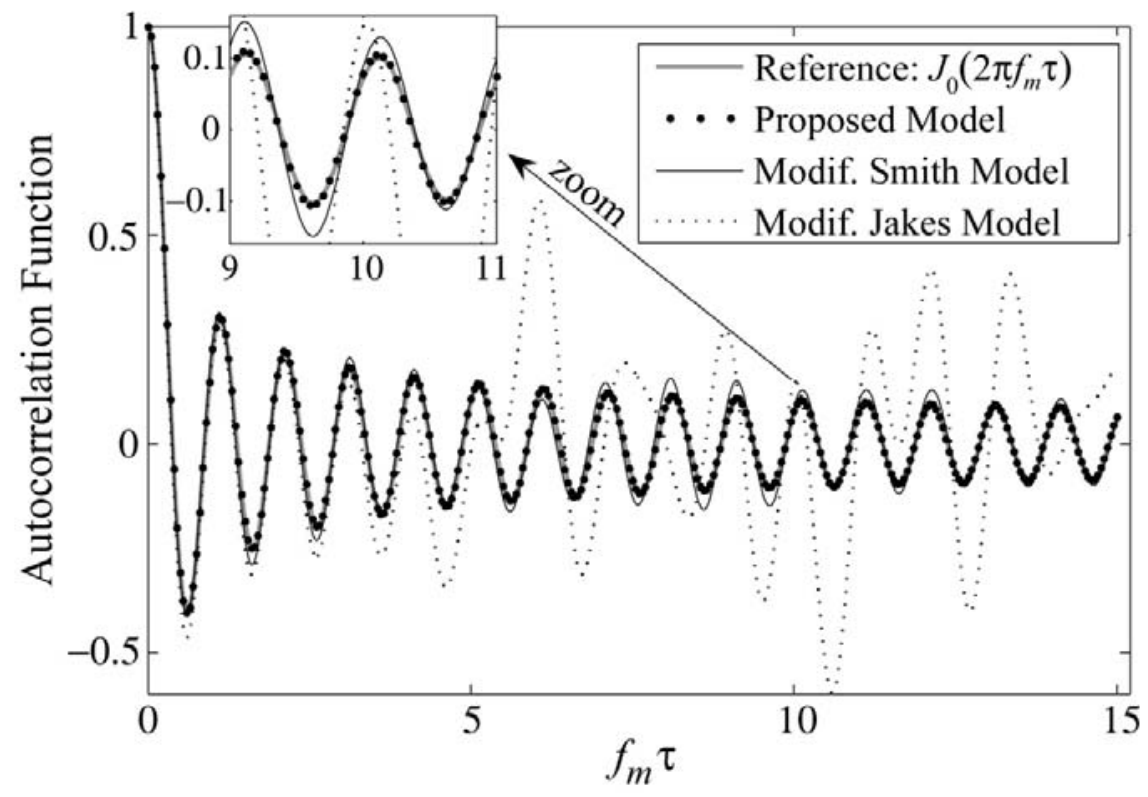


Figure 9 Normalised execution time (adopting as reference the Modif. Jakes greatest value) averaged over 30 simulations with a set of six sample functions

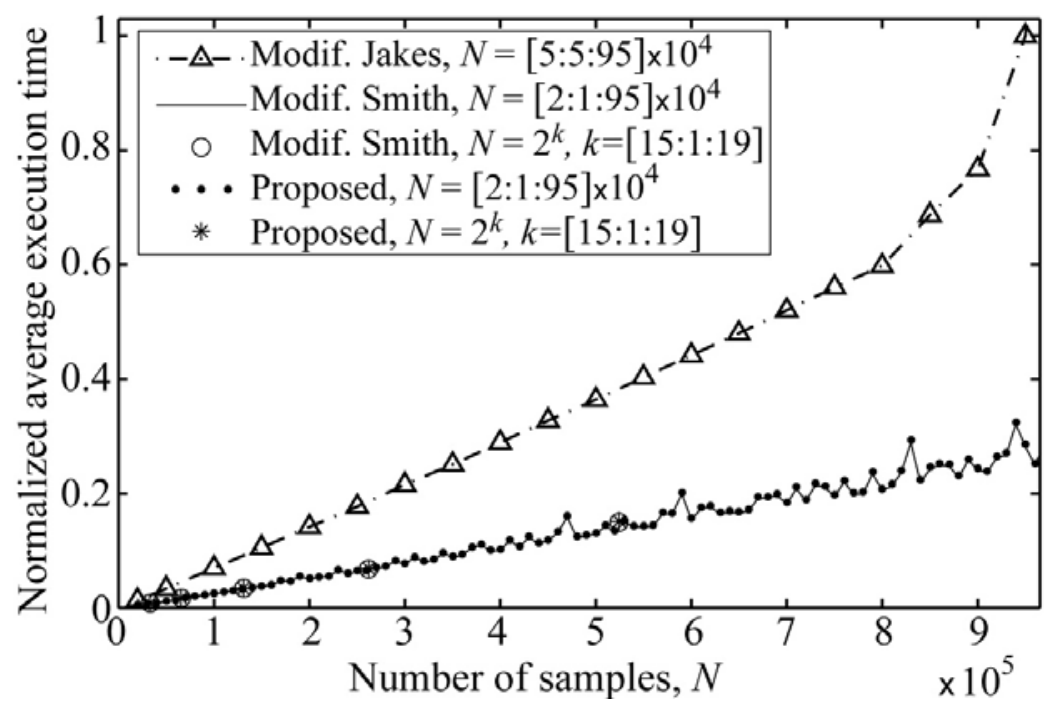

Note: $k$ and $N=$ [start:increment:end]

Figure 10 Cross-correlation between the real and imaginary parts of $c[n]$ generated with Clarke PSD

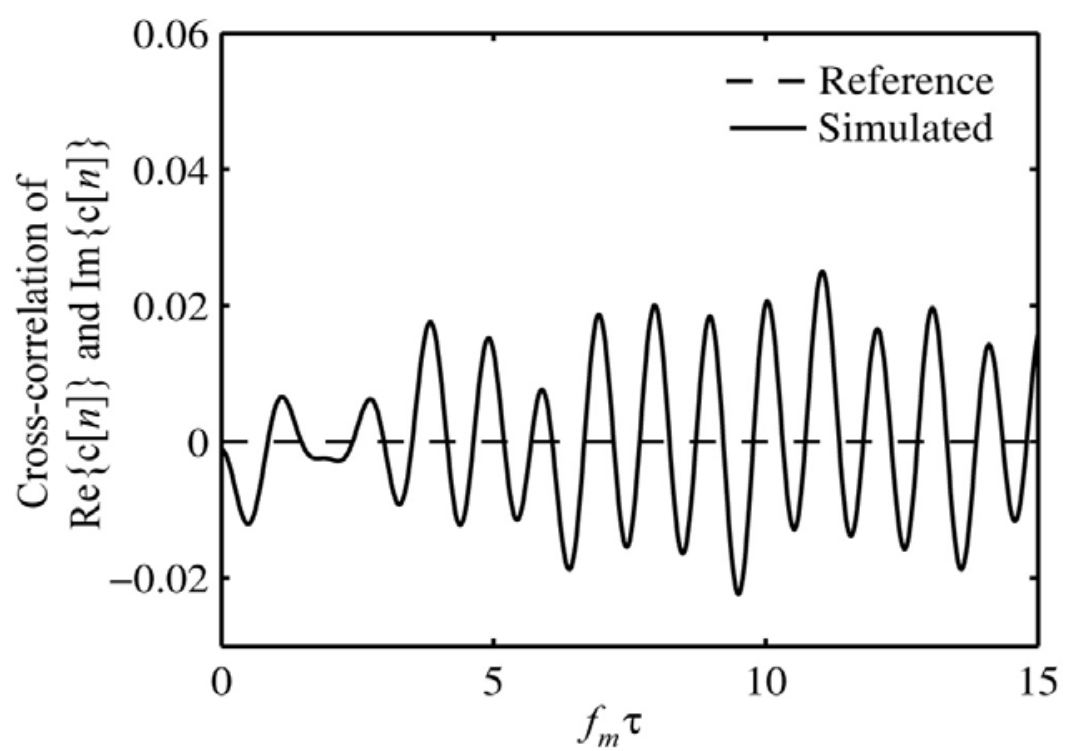


Figure 11 The autocorrelation of $c[n]$, generated with Aulin PSD, is compared with a reference calculated using (4) and the Bessel function of the first kind $J_{0}($.

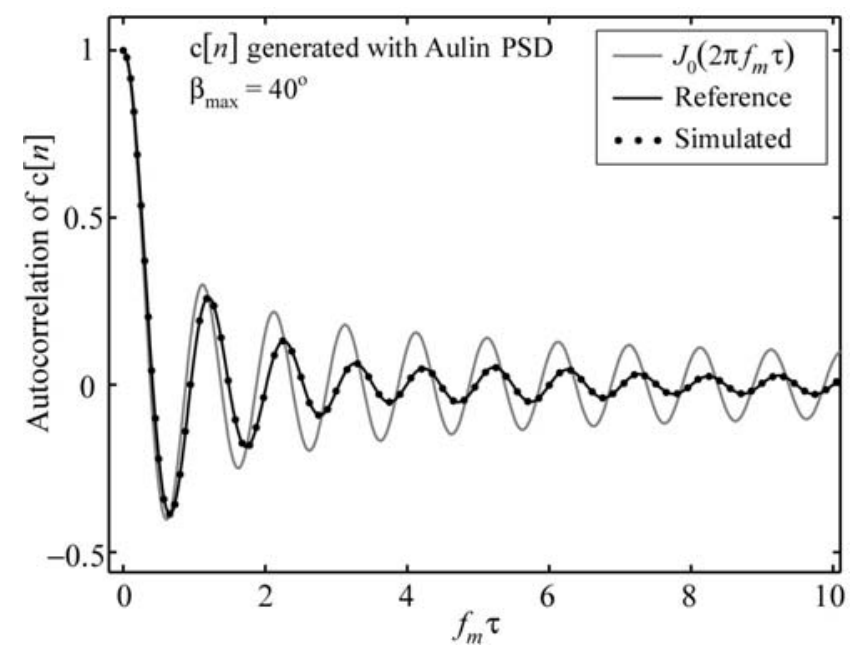

Figure 12 LCR and AFD (Clarke PSD)
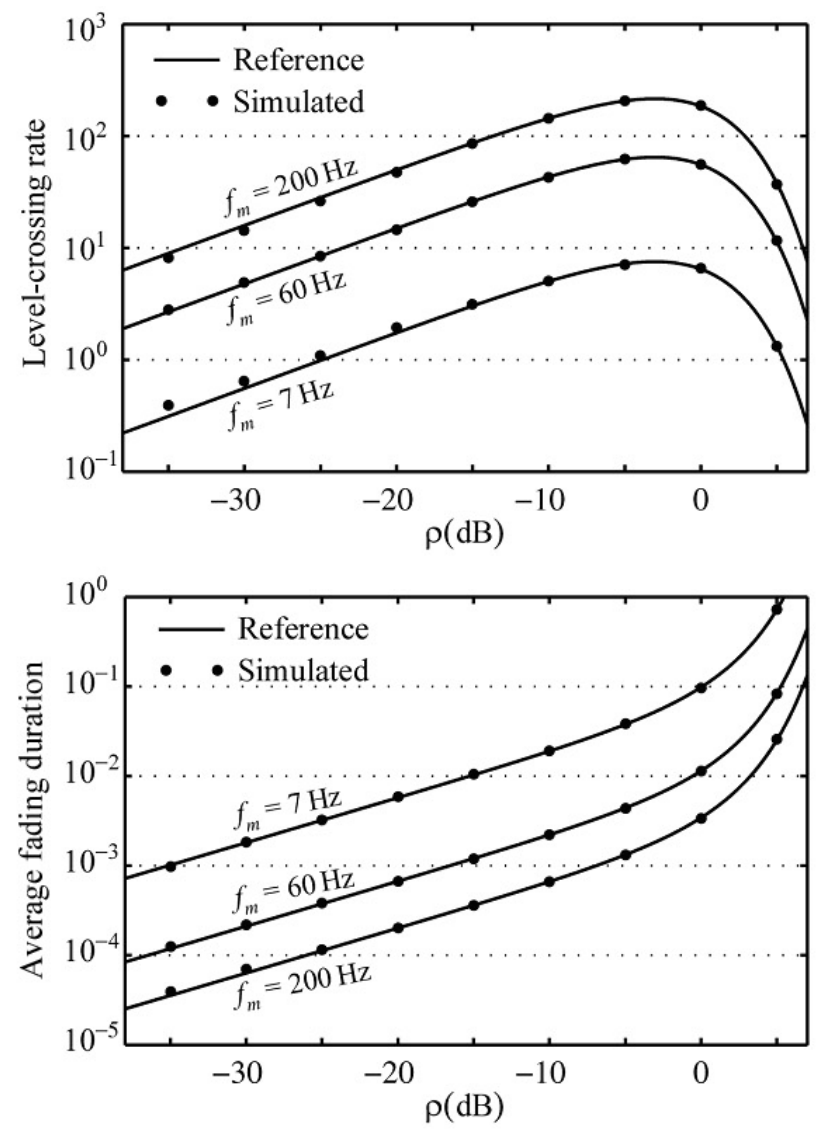
Figure 13 Cross-correlation of two sample functions generated with Clarke PSD

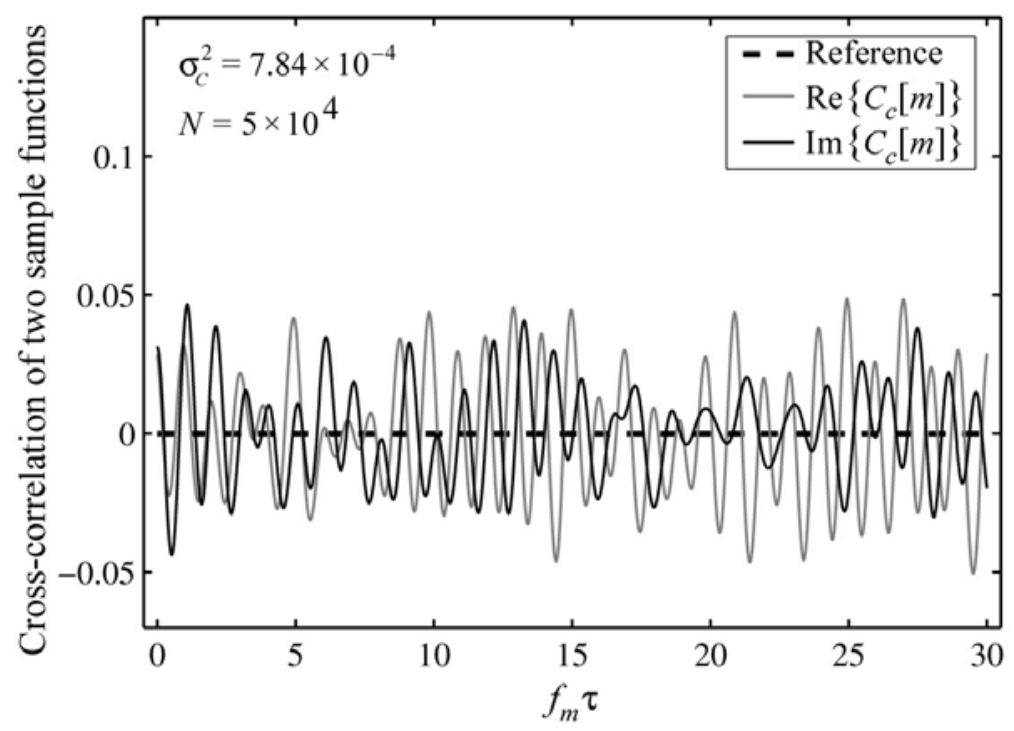

Figure 14 Variance of $C_{c}[m]$ i.e., $\sigma_{c}^{2}$ ) using Clarke and Aulin PSDs.

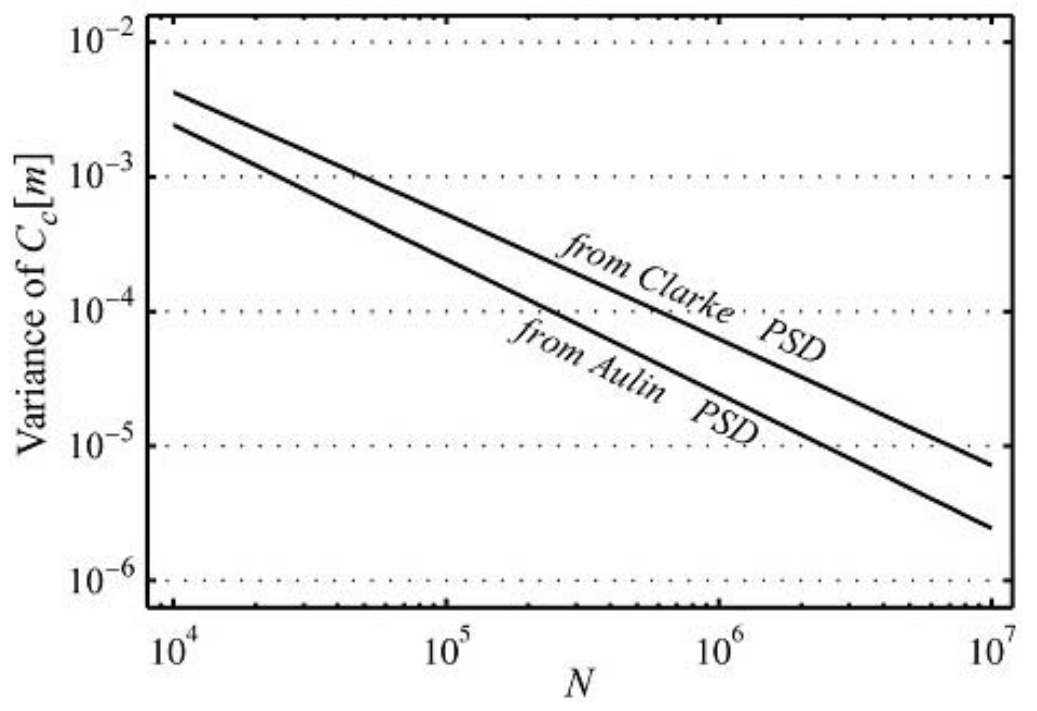

\section{Conclusions}

A new discrete-time statistical model for simulation of multiple uncorrelated Rayleigh waveforms was presented. The model and its statistical characteristics were derived as a function of the channel PSD. It was verified that, irrespective of the channel PSD shape, this model is ergodic, and its ensemble averages agree with 
those found in a physical mobile radio channel. This characteristic is of great importance for transmission coefficients generation where the PSD does not follow the traditional Clarke and Aulin shapes. In addition, only a single random number generator is required to generate the transmission coefficients, which is an advantage over the Smith (1975) model. However, the computational complexity in order to generate the sequence $e^{j \varphi k}$ in the proposed model is approximately twice as much as the necessary to generate $N$ complex Gaussian numbers reported in Young and Beaulieu (2000), resulting in a similar final computational complexity for the two models. Nevertheless, the proposed model is more general than the one presented in Young and Beaulieu (2000) because it can be used for every PSD shape.

Comparative results for Clarke PSD show that the IDFT models require smaller computational effort. Besides, the proposed model presents results slightly better than the modified Smith model concerning the autocorrelation function.

\section{Acknowledgements}

The authors would like to thank the anonymous reviewers for their comments, which helped to improve the presentation of this paper.

\section{References}

Alimohammad, A., Fard, S.F., Cockburn, B.F. and Schlegel, C. (2009) 'Compact Rayleigh and Rician fading simulator based on random walk processes', IET Communications, Vol. 3, No. 8, pp.1333-1342.

Aulin, T. (1979) 'A modified model for the fading signal at a mobile radio channel', Transactions on Vehicular Technology, August, Vol. VT-28, No. 3, pp.182-203.

Clarke, R.H. (1968) 'A statistical theory of mobile radio reception', Bell System Technical Journal, July, Vol. 47, pp.957-1000.

Cooley, J.W. and Tukey, J.W. (1965) 'An algorithm for the machine computation of complex Fourier series', Mathematics of Computation, April, Vol. 19, No. 90, pp.297-301.

Couch, L.W. II (2006) Digital and Analog Communication Systems, 7th ed., Prentice Hall, New Jersey.

Dent, P.G., Bottomley, E. and Croft, T. (1993) 'Jakes fading model revisited', Electronics Letters, June, Vol. 29, No. 13, pp.1162-1163.

Frigo, M. and Johnson, S.G. (1998) 'An adaptive software architecture for the FFT', Proc. Int. Conf. Acoustics, Speech, and Signal Processing, Vol. 3, pp.1381-1384.

Iqbal, R., Abhayapala, T.D. and Lamahewa, T.A. (2009) 'Generalised Clarke model for mobile-radio reception', IET Communications, Vol. 3, No. 4, pp.644-654.

Jakes, W.C. (1974) Microwave Mobile Communications, Wiley, New York.

Kodzovi, A. and Abdi, A. (2003) 'Efficient simulation of space-time correlated MIMO mobile fading channels', in Proc. IEEE Vehicular Technology Conference, Orlando, FL, October, pp.652-656.

Oppenheim, A.V., Schafer, R.W. and Buck, J.R. (1999) Discrete-Time Signal Processing, 2nd ed., Prentice Hall, New Jersey.

Papoulis, A. (2001) Probability, Random Variables, and Stochastic Process, 4th ed., McGraw-Hill, Inc, New York. 
Pop, M. and Beaulieu, N.C. (2001) 'Limitations of sum-of-sinusoids fading channel simulators', IEEE Transactions on Communications, April, Vol. 49, No. 4, pp.699-708.

Shinozuka, M. and Deodatis, G. (1991) 'Simulation of stochastic processes by spectral representation', Applied Mechanics Reviews, April, Vol. 44, No. 4, pp.191-203.

Silva, V., Abrão, T. and Jeszensky, P.J.E. (2004) 'Statistically correct simulation models for the generation of multiple uncorrelated Rayleigh fading waveforms', in 2004 IEEE International Symposium on Spread Spectrum Techniques and Applications, Sydney, Australia, September, pp.472-476.

Smith, J.I. (1975) 'A computer generating multipath fading simulation for mobile radio', IEEE Transactions on Vehicular Technology, August, Vol. VT-24, No. 3, pp.39-40.

Wang, C.X. and Pätzold, M. (2003) 'Methods of generating multiple uncorrelated Rayleigh fading processes', Proc. 57th IEEE Semiannual Vehicular Technology Conference, April, Vol. 1, pp.510-514.

Xiao, C., Zheng, Y.R. and Beaulieu, N.C. (2002) 'Second-order statistical properties of the WSS Jakes' fading channel simulator', IEEE Transactions on Communications, June, Vol. 50, No. 6, pp.888-891.

Young, D.J. and Beaulieu, N.C. (2000) 'The generation of correlated Rayleigh random variates by inverse discrete Fourier transform', IEEE Transactions on Communications, July, Vol. 48, No. 7, pp.1114-1127.

Zeng, Y. and Xiao, C. (2003) 'Simulation models with correct statistical properties for Rayleigh fading channels', IEEE Transactions on Communications, June, Vol. 51, No. 6 , pp.920-928.

\section{Appendix A}

\section{Ensemble averages of $c[n]$}

a mean value

$$
\begin{gathered}
E\{\mathrm{c}[n]\}=\sqrt{\frac{2 \sigma^{2}}{N}} \sum_{k=0}^{N-1} \sqrt{S[k]} e^{j(2 \pi k n / N)} E\left\{e^{j \varphi_{k}}\right\}=0 \\
\Rightarrow E\{\operatorname{Re}\{\mathrm{c}[n]\}\}=E\{\operatorname{Im}\{\mathrm{c}[n]\}\}=0
\end{gathered}
$$

b autocorrelation function

$$
\begin{aligned}
& R\left[n_{1}, n_{2}\right]=E\left\{c^{*}\left[n_{1}\right] c\left[n_{2}\right]\right\} \\
& =\frac{2 \sigma^{2}}{N}\left(\sum_{\substack{k=0 \\
k \neq z}}^{N-1} \sum_{z=0}^{N-1} \sqrt{S[k] S[z]} e^{+j\left(2 \pi\left(z n_{2}-k n_{1}\right) / N\right)} E\left\{e^{+j \varphi_{z}}\right\} E\left\{e^{-j \varphi_{k}}\right\}+\right. \\
& \left.\sum_{k=0}^{N-1} S[k] e^{+j\left(2 \pi k\left(n_{2}-n_{1}\right) / N\right)} E\left\{e^{+j\left(\varphi_{k}-\varphi_{k}\right)}\right\}\right) \\
& =\frac{2 \sigma^{2}}{N} \sum_{k=0}^{N-1} S[k] e^{+j(2 \pi k m / N)}, \quad \text { where } \quad m=n_{2}-n_{1} \\
& \Rightarrow R[m]=2 \sigma^{2} \operatorname{IDFT}\{S[k]\}, \quad 0 \leq m<N
\end{aligned}
$$


c autocorrelation function of $\operatorname{Re}\{c[n]\}$

$$
\begin{aligned}
& g_{R}\left[n_{1}, n_{2}\right]=E\left\{\operatorname{Re}\left\{c\left[n_{1}\right]\right\} \operatorname{Re}\left\{c\left[n_{2}\right]\right\}\right\} \\
& =\frac{1}{4} E\left\{\left(c\left[n_{1}\right]+c^{*}\left[n_{1}\right]\right)\left(c\left[n_{2}\right]+c^{*}\left[n_{2}\right]\right)\right\} \\
& =\frac{\sigma^{2}}{2} \frac{1}{N} \sum_{k=0}^{N-1} S[k]\left(e^{j(2 \pi k m / N)}+e^{-j(2 \pi k m / N)}\right) \\
& =\frac{1}{2} \sigma^{2}\left(\operatorname{IDFT}\{S[k]\}+(\operatorname{IDFT}\{S[k]\})^{*}\right) \\
& =\sigma^{2} \operatorname{Re}\{\operatorname{IDFT}\{S[k]\}\}, \quad 0 \leq m<N
\end{aligned}
$$

where $m=n_{2}-n_{1}$. For $S[k]$ real and even (as Clarke model), $g_{R}\left[n_{1}, n_{2}\right]=g[m]=\sigma^{2} \operatorname{IDFT}\{\mathrm{S}[\mathrm{k}]\}$.

d Autocorrelation function of $\operatorname{Im}\{c[n]\}$

$$
\begin{aligned}
& g_{I}\left[n_{1}, n_{2}\right]=E\left\{\operatorname{Im}\left\{c\left[n_{1}\right]\right\} \operatorname{Im}\left\{c\left[n_{2}\right]\right\}\right\} \\
& =\frac{1}{4} E\left\{\left(c\left[n_{1}\right]-c^{*}\left[n_{1}\right]\right)\left(c^{*}\left[n_{2}\right]-c\left[n_{2}\right]\right)\right\} \\
& =\frac{\sigma^{2}}{2 N}\left(\sum _ { \substack { k = 0 \\
k \neq z } } ^ { N - 1 N - 1 } \left(\sqrt{S[k] S[z]} E\left\{e^{j\left(\varphi_{k}+2 \pi k n_{1} / N\right)}-e^{-j\left(\varphi_{k}+2 \pi k n_{1} / N\right)}\right\}\right.\right. \\
& \left.E\left\{e^{-j\left(\varphi_{z}+2 \pi z n_{2} / N\right)}-e^{j\left(\varphi_{z}+2 \pi z n_{2} / N\right)}\right\}\right)+\sum_{k=0}^{N-1}(S[k] \\
& \left.\left.E\left\{\left(e^{j\left(\varphi_{k}+2 \pi k n_{1} / N\right)}-e^{-j\left(\varphi_{k}+2 \pi k n_{1} / N\right)}\right)\left(e^{-j\left(\varphi_{k}+2 \pi k n_{2} / N\right)}-e^{j\left(\varphi_{k}+2 \pi k n_{2} / N\right)}\right)\right\}\right)\right) \\
& =\frac{\sigma^{2}}{2 N} \sum_{k=0}^{N-1} S[k]\left(e^{j\left(2 \pi k\left(n_{2}-n_{1}\right) / N\right)}+e^{-j\left(2 \pi k\left(n_{2}-n_{1}\right) / N\right)}\right) \\
& =g_{R}\left[n_{1}, n_{2}\right]
\end{aligned}
$$

e cross-correlation of $\operatorname{Re}\{c[n]\}$ and $\operatorname{Im}\{c[n]\}$

$$
\begin{aligned}
& h\left[n_{1}, n_{2}\right]=E\left\{\operatorname{Re}\left\{c\left[n_{1}\right]\right\} \operatorname{Im}\left\{c\left[n_{2}\right]\right\}\right\} \\
& =j \frac{1}{4}\left(R[-m]-R[m]+E\left\{c^{*}\left[n_{1}\right] c^{*}\left[n_{2}\right]-c\left[n_{1}\right] c\left[n_{2}\right]\right\}\right)
\end{aligned}
$$

But, $E\left\{c^{*}\left[n_{1}\right] c^{*}\left[n_{2}\right]\right\}=0$ and $E\left\{c\left[n_{1}\right] c\left[n_{2}\right]\right\}=0$. Then,

$$
\begin{aligned}
& h[m]=j \frac{1}{4}(R[-m]-R[m]) \\
& =j \frac{1}{4}\left(2 \sigma^{2}(\operatorname{IDFT}\{S[k]\})^{*}-2 \sigma^{2} \operatorname{IDFT}\{S[k]\}\right) \\
& =\sigma^{2} \operatorname{Im}\{\operatorname{IDFT}\{S[k]\}\}, \quad 0 \leq m<N
\end{aligned}
$$


For $S[k]$ real and even, $h[m]=0$.

In summary, the ensemble averages agree with (19).

\section{Appendix B}

\section{Time averages of $c[n]$}

Let $c_{a}[n]$ be the $a$ th sample function of the random process $c[n]$.

a time average

$$
\begin{aligned}
& \left\langle\left[c_{a}[n]\right]\right\rangle=\lim _{N \rightarrow \infty} \frac{1}{N} \sum_{n=0}^{N-1} c_{a}[n] \\
& =\lim _{N \rightarrow \infty} \sqrt{\frac{2 \sigma^{2}}{N} \sum_{k=0}^{N-1} \sqrt{S[k]}} e^{j \varphi_{a, k}} \frac{1}{N} \sum_{n=0}^{N-1} e^{j 2 \pi k n / N} \\
& =\lim _{N \rightarrow \infty} \sqrt{\frac{2 \sigma^{2}}{N} S[0] e^{j \varphi_{a, 0}}}=0
\end{aligned}
$$

b time autocorrelation function

$$
\begin{array}{r}
\left\langle\left[c_{a}^{*}[n] c_{a}[n+m]\right]\right\rangle=\lim _{N \rightarrow \infty} \frac{1}{N} \sum_{n=0}^{N-1}\left[c_{a}^{*}[n] c_{a}[n+m]\right] \\
=\lim _{N \rightarrow \infty} \frac{2 \sigma^{2}}{N} \sum_{k=0}^{N-1} \sum_{z=0}^{N-1}\left(\sqrt{S[k]} \sqrt{S[z]} e^{j\left(\varphi_{a, z}-\varphi_{a, k}\right)} e^{j 2 \pi z m / N}\right. \\
\left.\frac{1}{N} \sum_{n=0}^{N-1} e^{j 2 \pi(z-k) n / N}\right) \\
=\lim _{N \rightarrow \infty} \frac{2 \sigma^{2}}{N} \sum_{k=0}^{N-1} S[k] e^{j 2 \pi k m / N}=\lim _{N \rightarrow \infty} 2 \sigma^{2} \operatorname{IDFT}\{S[k]\}
\end{array}
$$

For large $N,\left\langle\left[c_{a}^{*}[n] c_{a}[n+m]\right]\right\rangle \cong R[m]$.

c time cross-correlation of $\operatorname{Re}\left\{c_{a}[n]\right\}$ and $\operatorname{Im}\left\{c_{a}[n]\right\}$

$$
\begin{aligned}
& \left\langle\left[\operatorname{Re}\left\{c_{a}[n]\right\} \operatorname{Im}\left\{c_{a}[n+m]\right\}\right]\right\rangle \\
& =\lim _{N \rightarrow \infty} j \frac{1}{4 N} \sum_{n=0}^{N-1}\left[\left(c_{a}^{*}[n]+c_{a}[n]\right)\left(c_{a}^{*}[n+m]-c_{a}[n+m]\right)\right] \\
& =\lim _{N \rightarrow \infty} j \frac{\sigma^{2}}{2 N} \sum_{k=0}^{N-1} S[k]\left(e^{-j(2 \pi k m / N)}-e^{j(2 \pi k m / N)}\right) \\
& =\lim _{N \rightarrow \infty} \sigma^{2} \operatorname{Im}\{\operatorname{IDFT}\{S[k]\}\}
\end{aligned}
$$


For large $N,\left\langle\left[\operatorname{Re}\left\{c_{a}[n]\right\} \operatorname{Im}\left\{c_{a}[n+m]\right\}\right]\right\rangle \cong h[m]$.

d time autocorrelation of $\operatorname{Re}\left\{c_{a}[n]\right\}$

$$
\begin{aligned}
& \left\langle\left[\operatorname{Re}\left\{c_{a}[n]\right\} \operatorname{Re}\left\{c_{a}[n+m]\right\}\right]\right\rangle \\
& =\lim _{N \rightarrow \infty} \frac{1}{4 N} \sum_{n=0}^{N-1}\left[\left(c_{a}^{*}[n]+c_{a}[n]\right)\left(c_{a}^{*}[n+m]+c_{a}[n+m]\right)\right] \\
& =\lim _{N \rightarrow \infty} \frac{\sigma^{2}}{2 N} \sum_{k=0}^{N-1} S[k]\left(e^{-j(2 \pi k m / N)}+e^{j(2 \pi k m / N)}\right) \\
& =\lim _{N \rightarrow \infty} \sigma^{2} \operatorname{Re}\{\operatorname{IDFT}\{S[k]\}\}
\end{aligned}
$$

For large $N,\left\langle\left[\operatorname{Re}\left\{c_{a}[n]\right\} \operatorname{Re}\left\{c_{a}[n+m]\right\}\right]\right\rangle \cong g[m]$.

e time autocorrelation of $\operatorname{Im}\left\{c_{a}[n]\right\}$

$$
\begin{aligned}
& \left\langle\left[\operatorname{Im}\left\{c_{a}[n]\right\} \operatorname{Im}\left\{c_{a}[n+m]\right\}\right]\right\rangle \\
& =\lim _{N \rightarrow \infty} \frac{1}{4 N} \sum_{n=0}^{N-1}\left[\left(c_{a}[n]-c_{a}^{*}[n]\right)\left(c_{a}^{*}[n+m]-c_{a}[n+m]\right)\right] \\
& =\lim _{N \rightarrow \infty} \frac{\sigma^{2}}{2 N} \sum_{k=0}^{N-1} S[k]\left(e^{-j(2 \pi k m / N)}+e^{j(2 \pi k m / N)}\right) \\
& =\lim _{N \rightarrow \infty} \sigma^{2} \operatorname{Re}\{\operatorname{IDFT}\{S[k]\}\}
\end{aligned}
$$

For large $N,\left\langle\left[\operatorname{Im}\left\{c_{a}[n]\right\} \operatorname{Im}\left\{c_{a}[n+m]\right\}\right]\right\rangle \cong g[m]$.

f time cross-correlation of two sample functions

$$
\begin{aligned}
& C_{c}[m]=\left\langle\left[c_{a}^{*}[n] c_{b}[n+m]\right]\right\rangle=\lim _{N \rightarrow \infty} \frac{1}{N} \sum_{n=0}^{N-1}\left[c_{a}^{*}[n] c_{b}[n+m]\right] \\
& =\lim _{N \rightarrow \infty} \frac{2 \sigma^{2}}{N} \sum_{k=0}^{N-1} \sum_{z=0}^{N-1}\left(\sqrt{S[k]} \sqrt{S[z]} e^{j\left(\varphi_{b, z}-\varphi_{a, k}\right)} e^{j 2 \pi z m / N}\right. \\
& =\lim _{N \rightarrow \infty} \frac{2 \sigma^{2}}{N} \sum_{n=0}^{N-1} e^{j 2 \pi(z-k) n / N} \sum_{k=0}^{N-1}\left(\sqrt{S[k]} \sqrt{S[z]} e^{j\left(\varphi_{b, z}-\varphi_{a, k}\right)} e^{j 2 \pi z m / N}\right) \\
& =\lim _{N \rightarrow \infty} \frac{2 \sigma^{2}}{N} \sum_{k=0}^{N-1} S[k] e^{j\left(\varphi_{b, k}-\varphi_{a, k}\right)} e^{j 2 \pi k m / N}
\end{aligned}
$$

So the mean and variance of $C_{c}[m]$ are given by 


$$
\begin{aligned}
& E\left\{C_{c}[m]\right\}=\lim _{N \rightarrow \infty} \frac{2 \sigma^{2}}{N} \sum_{k=0}^{N-1} S[k] E\left\{e^{j\left(\varphi_{b, k}-\varphi_{a, k}\right)}\right\} e^{j 2 \pi k m / N}=0 \\
& \sigma_{C}^{2}=E\left\{C_{c}[m] C_{c}^{*}[m]\right\}=E\left\{\lim _{N \rightarrow \infty}\left(\frac{2 \sigma^{2}}{N}\right)^{2} \sum_{k=0}^{N-1} \sum_{z=0}^{N-1}(S[k] S[z]\right. \\
& \left.\left.=\lim _{N \rightarrow \infty}\left(\frac{2 \sigma^{2}}{N}\right)^{2} \sum_{k=0}^{N-1} \sum_{z=0}^{N-1}\left(S[k] S[z] e^{j 2 \pi(k-z) m / N}-\varphi_{a, z}\right) e^{-j 2 \pi z m / N} e^{j\left(\varphi_{b, k}-\varphi_{a, k}\right)} e^{j 2 \pi k m / N}\right)\right\} \\
& =\lim _{N \rightarrow \infty} \frac{\left(2 \sigma^{2}\right)^{2}}{N^{2}} \sum_{k=0}^{N-1} S[k]^{2}=0 \\
& \Rightarrow C_{c}[m] \cong 0, \quad \text { for large } N .
\end{aligned}
$$

\section{Appendix C}

\section{Interpolation procedure}

Let $A_{m}$ be the area below the curve $\mathrm{S}(f)$ from $\left(k_{m}-1\right)$ to $k_{m} f_{\Delta}$ as shown in Figure $\mathrm{C}(1)$. Considering the area from 0 to $k_{m} f_{\Delta}$ approximately $1 / 2$ [from (11)] and approximating $A_{m}$ by a rectangle of height $S\left(k_{m} f_{\Delta}\right)$ and width $f_{\Delta}$, the following relation can be obtained for $k=k_{m}$

$$
\frac{1}{2}-\int_{0}^{\left(k_{m}-1\right) f_{\Delta}} S(f) d f \cong S\left(k_{m} f_{\Delta}\right) f_{\Delta}
$$

Now, one can show by using and solving $(\mathrm{C} 1) S\left(k_{m} f_{\Delta}\right)$ that

$$
S\left(k_{m} f_{\Delta}\right) \cong \frac{1}{f_{\Delta}}\left(\frac{1}{2}-\frac{1}{\pi} \arcsin \left(\frac{\left(k_{m}-1\right) f_{\Delta}}{f_{m}}\right)\right)
$$

and, substituting $S\left[k_{m}\right] T_{S}=S\left(k_{m} f_{\Delta}\right)$ in (C2), one can obtain $S\left[k_{m}\right]$ in (22).

Similar results (based in the exact area from 0 to $k_{m} f_{\Delta}$ ) are mentioned in Smith (1975) and Young and Beaulieu (2000) for the magnitude spectrum. 
Figure C1 Interpolation procedure for the Clarke PSD

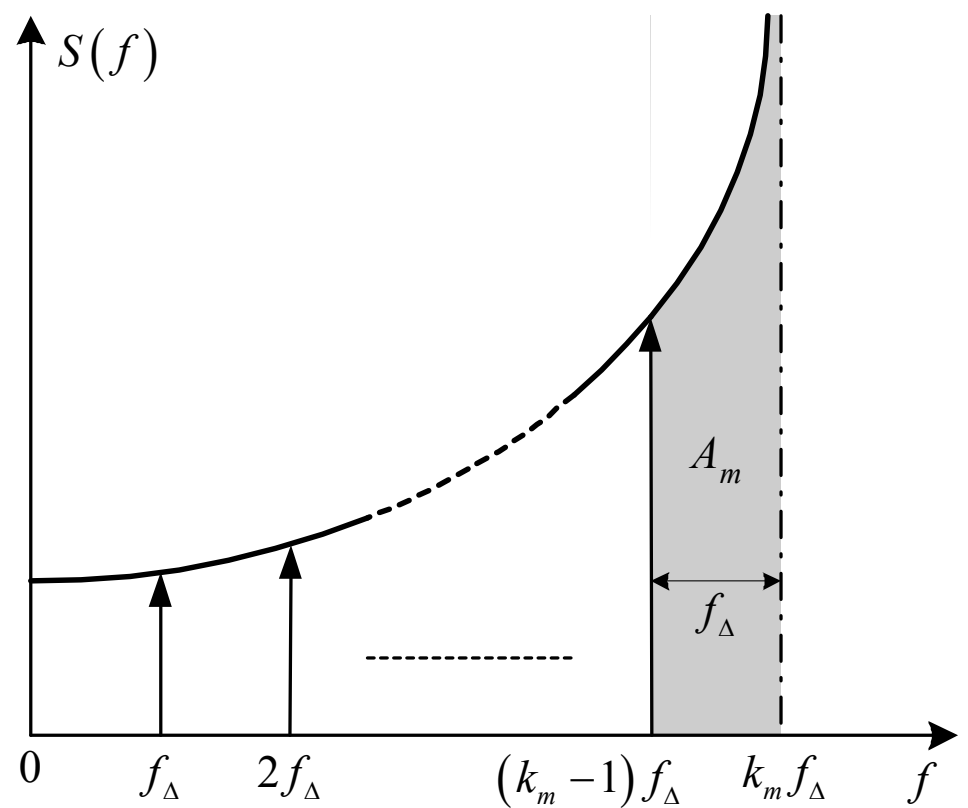

In fact, the range of $k_{m} f_{\Delta}$ can be obtained from (23): $f_{m}-f_{\Delta}<k_{m} f_{\Delta} \leq f_{m}$. In practice, the difference between $k_{m} f_{\Delta}$ and $f_{m}$ is negligible. 


\section{Appendix D}

\section{List of mathematical notation}

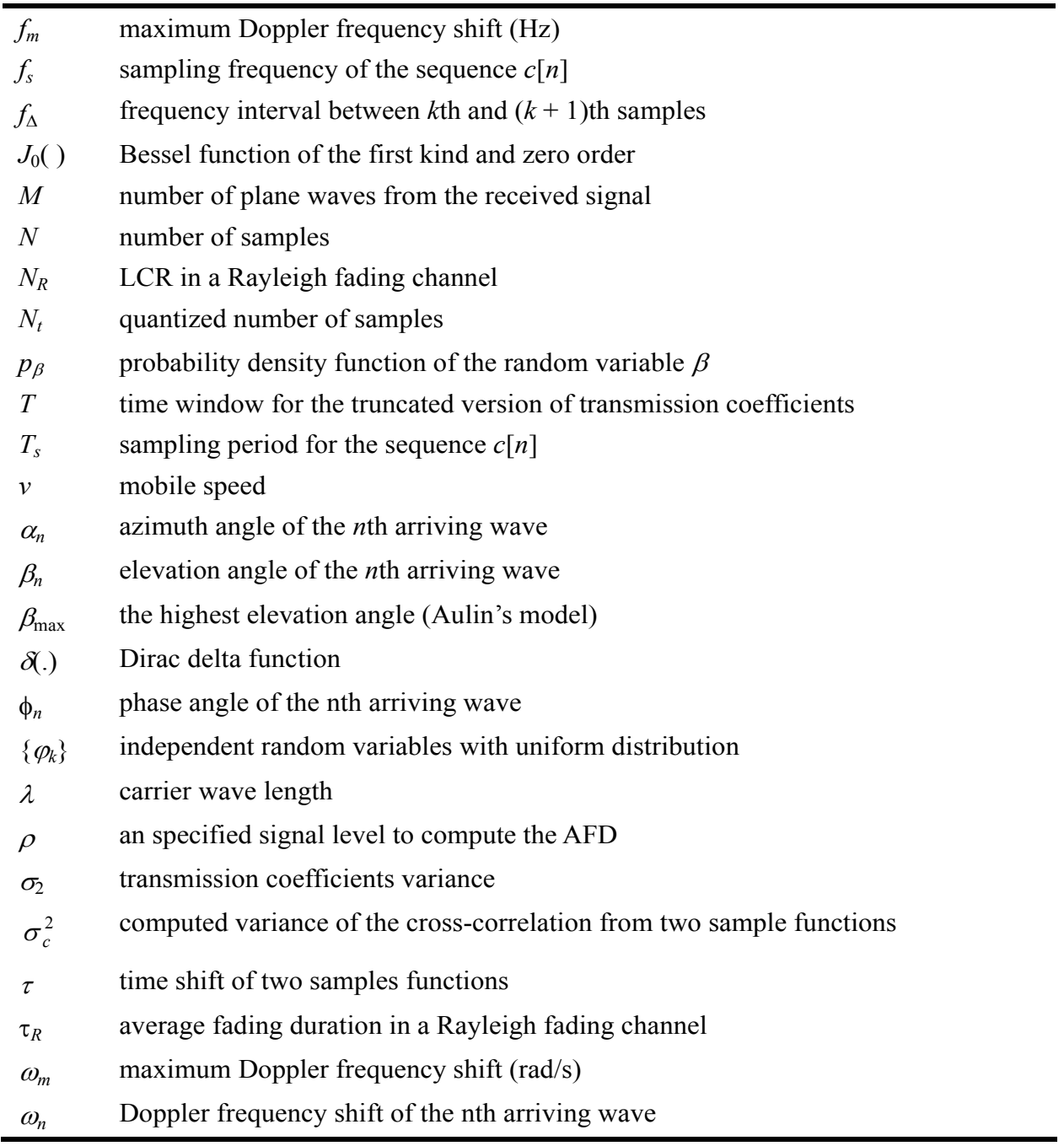

